\title{
IDENTIFICAÇÃO E IMPLICAÇÕES PARA A CONSERVAÇÃO DO SOLO DAS FONTES DE SEDIMENTOS EM BACIAS HIDROGRÁFICAS ${ }^{(1)}$
}

\author{
Jean Paolo Gomes Minella ${ }^{(2)}$, Gustavo Henrique Merten ${ }^{(3)}$, José \\ Miguel Reichert $^{(4)}$ \& Danilo Rheinheimer dos Santos ${ }^{(4)}$
}

\begin{abstract}
RESUMO
Os sedimentos oriundos de áreas agrícolas são importante fonte de poluentes para os ecossistemas aquáticos. Existem várias fontes de sedimentos na escala de uma bacia hidrográfica rural, como lavouras, pastagens, florestas, rede fluvial e estradas. Cada uma tem características diferentes na magnitude de contribuição e potencial de contaminação. Identificar a origem dos sedimentos é fundamental na compreensão da taxa de emissão de sedimentos e no manejo dos sedimentos erodidos na escala de bacia. Este trabalho foi desenvolvido com o propósito de identificar as principais fontes de sedimentos de duas bacias rurais de cabeceira no Sul do Brasil, as quais são representativas do sistema agrário e das características fisiográficas da região. A metodologia utiliza a comparação entre os solos de diferentes fontes e os sedimentos em suspensão, usando traçadores naturais. A análise discriminante encontrou um grupo de variáveis capazes de distinguir as fontes de sedimentos. Com base na análise de classificação, as contribuições relativas de cada fonte nas bacias de Agudo e Arvorezinha foram, respectivamente: lavouras $(68,3$ e $55,5 \%)$, estradas $(28,1$ e $37,6 \%)$ e rede fluvial $(3,6$ e $6,9 \%)$. Os resultados mostram que, além da contribuição predominante das lavouras, as erosões nas estradas e na rede fluvial contribuem significativamente para produção total de sedimentos nas bacias, demonstrando a importância dessa informação para controle da erosão do solo e manejo dos sedimentos na escala de bacia hidrográfica.
\end{abstract}

Termos de indexação: erosão hídrica, traçadores naturais, produção de sedimentos.

\footnotetext{
(1) Trabalho desenvolvido com o apoio Conselho de Desenvolvimento Científico e Tecnológico. Recebido para publicação em outubro de 2006 e aprovado em outubro de 2007.

${ }^{(2)}$ Doutorando do Instituto de Pesquisas Hidráulicas, Universidade Federal do Rio Grande do Sul - UFRGS. Av. Bento Gonçalves 9500, CEP 91501-970 Caixa Postal 15029 - Porto Alegre (RS). E-mail: jminella@ppgiph.ufrgs.br

(3) Professor do Instituto de Pesquisas Hidráulicas, UFRGS. E-mail: merten@iph.ufrgs.br

(4) Professor do Departamento de Solos, Centro de Ciências Rurais, Universidade Federal de Santa Maria - UFSM. Camobi, CEP 97105-900 Santa Maria (RS). E-mails: reichert@smail.ufsm.br; danilo@ccr.ufsm.br
} 


\title{
SUMMARY: IDENTIFICATIONAND IMPLICATIONS FOR SOIL CONSERVATION OF SEDIMENT SOURCES IN CATCHMENT AREAS
}

\begin{abstract}
Sediments in agricultural areas represent a significant source of pollution of aquatic environments. There are many different sediment sources at the level of a rural catchment (e.g. crop fields, pastures, forests, river banks, and roads), with different magnitude of contribution and contaminant potential. The identification of the sediment origin is important to understand the sediment transport rate and the management of sediment eroded at the catchment scale. The goal of this work was to identify the main sediment sources of two rural headwater catchments in southern Brazil, representative of the regional agrarian system and physiographic characteristics. The methodology was based on a comparison between soils of different sources and the eroded sediments using natural tracers. A set of variables was found by the discriminant soil analysis to distinguish the sources. Based on the classification analysis, the relative contribution of each source to the eroded sediments in the Agudo and Arvorezinha catchments was, respectively: crop fields 68.3 and $55.5 \%$, roads 28.1 and $37.6 \%$ and bank erosion 3.6 and $6.9 \%$. Results show that besides the major contribution coming from crop fields, erosion from roads and drainage represents a significant part of the total sediment. Such information is essential to control soil erosion and for sediment management at the catchment scale.
\end{abstract}

Index terms: water erosion, natural tracers, sediment production.

\section{INTRODUÇÃO}

No Sul do Brasil, uma parcela significativa da agricultura familiar ocupa áreas de baixa aptidão agrícola e alta fragilidade ambiental (declividade acentuada das vertentes e solos rasos). A erosão hídrica é a principal conseqüência dessa condição, que causa redução da capacidade produtiva dos solos e aumento da transferência de sedimentos e poluentes para os corpos de água (Merten \& Minella, 2003).

Agências governamentais têm implementado programas voltados para a conservação dos solos, por meio do fomento ao cultivo mínimo e ao uso de culturas de cobertura. Entretanto, um dos desafios consiste em avaliar a eficiência das práticas recomendadas em reduzir as taxas de erosão, considerando a escala de uma bacia hidrográfica. Para esse propósito, têm sido conduzidos projetos de monitoramento cujo objetivo é o de avaliar como e em quanto tempo as práticas de conservação do solo adotadas pelos agricultores são capazes de atenuar a erosão do solo. No monitoramento, os efeitos das práticas conservacionistas sobre o controle da erosão são avaliados por meio da variabilidade da produção de sedimentos, que, por sua vez, consiste na integração dos fluxos de sedimentos em suspensão, dentro de um intervalo de tempo.

O fluxo de sedimentos em suspensão nos rios é formado por uma mistura de sedimentos provenientes de diferentes fontes, cuja identificação permite avaliar as implicações das práticas conservacionistas sobre a erosão hídrica. Além disso, a identificação das fontes de sedimentos é importante para possibilitar a validação de modelos matemáticos distribuídos de produção de sedimentos (Walling et al., 2003).

As fontes de sedimentos podem ser determinadas em função de características químicas e físicas dos solos e dos elementos que são utilizados como propriedades traçadoras (Walling \& Woodward, 1992; Minella, 2003). Por meio da coleta dos sedimentos em suspensão, é possível comparar suas características com as fontes e, conseqüentemente, determinar a origem destas (Yu \& Oldfield, 1989). A comparação dos sedimentos coletados na rede fluvial com as fontes é realizada sob rigoroso procedimento estatístico, em que é selecionado o melhor conjunto de características químicas que apresentam capacidade discriminante entre as fontes (Collins et al., 1997).

Assim, uma etapa importante da aplicação desse método consiste em selecionar corretamente o grupo de variáveis discriminantes que são distintas para cada local (Collins \& Walling, 2002). Exemplo de aplicação bem sucedida desse método é citado por Collins et al. (1997), os quais determinaram a variabilidade das fontes de sedimentos em duas bacias no Reino Unido. O método permitiu a verificação da contribuição das áreas de cultivo, das pastagens, das florestas e da erosão na rede fluvial. Também Russel et al. (2001) obtiveram bons resultados com esse método aplicado em duas bacias rurais submetidas a intenso uso agrícola. Nesse estudo, os autores verificaram que os sedimentos transportados em suspensão apresentavam como fonte de origem a erosão laminar nas lavouras (34 e $65 \%$ ), erosão nos drenos (27 e $55 \%$ ) e erosão na rede fluvial (menos de $10 \%$ ). 
Muitos estudos de identificação de fontes têm sido utilizados em programas nacionais de manejo de bacias nos países da Europa e da Ásia e na Austrália (Walling, 2005). No Brasil, apesar dos crescentes problemas associados à erosão hídrica e presença de sedimentos e poluentes nos rios, os estudos de identificação de fontes são incipientes.

Este trabalho foi realizado com o propósito de identificar a contribuição das principais fontes de sedimentos de duas pequenas bacias hidrográficas rurais, utilizando um método que se baseia no uso de traçadores naturais.

\section{MATERIAL E MÉTODOS}

\section{Áreas de estudo}

As duas áreas estudadas encontram-se no Sul do Brasil, Estado do Rio Grande do Sul, nos municípios de Agudo $\left(29^{\circ} 38^{\prime} \mathrm{S}\right.$ e $\left.53^{\circ} 21^{\prime} \mathrm{O}\right)$ e Arvorezinha $\left(28^{\circ} 52^{\prime} \mathrm{S}\right.$ e $\left.52^{\circ} 05^{\prime} \mathrm{O}\right)$, região central e centro-norte do Estado, respectivamente (Figura 1). A bacia de Agudo apresenta área de drenagem de $1,68 \mathrm{~km}^{2}$, e a de Arvorezinha, de $1,19 \mathrm{~km}^{2}$. O clima nas duas bacias é classificado, segundo Köppen, como Cfb, subtropical úmido com verões quentes, inverno com geadas freqüentes e precipitação bem distribuída no ano.

A formação geológica da bacia de Arvorezinha é caracterizada por derrame basáltico, com presença de diques e sills de diabásio, compondo o Grupo São Bento da Formação Serra Geral. O relevo é declivoso (declividade média de $7 \mathrm{~cm} \mathrm{~m}^{-1}$ ), com vertentes curtas e vales encaixados. Os solos predominantes são Alissolo Crômico, Cambissolo Háplico e Neossolo Litólico. A precipitação média anual na área é de $1.600 \mathrm{~mm}$, enquanto as vazões média e máxima são de 0,4 e $1,6 \mathrm{~m}^{3} \mathrm{~s}^{-1}$, respectivamente, e o tempo de concentração, de $30 \mathrm{~min}$.
A bacia de Agudo é caracterizada pela presença de rochas vulcânicas ácidas e básicas da Formação Serra Geral. O relevo é declivoso, com declividade média de $12 \mathrm{~cm} \mathrm{~m}^{-1}$. Os solos predominantes são Chernossolo e Neossolo Litólico. A precipitação média anual na bacia é de $1.500 \mathrm{~mm}$, a vazão média e a máxima dos eventos são de 1,2 e $2,2 \mathrm{~m}^{3} \mathrm{~s}^{-1}$, respectivamente, e o tempo de concentração, de 21 min.

As duas áreas são representativas do sistema agrícola e agrário das pequenas propriedades familiares do Sul do Brasil, voltado para produção de fumo. O uso predominante do solo é o cultivo de fumo; as lavouras, nas bacias de Agudo e Arvorezinha, ocupam área de 67 e 53 ha, respectivamente. O manejo do solo para cultivo do fumo, bem como as condições de precipitação, são considerados os fatores controladores da variabilidade intra-anual da produção de sedimentos em ambas as áreas. O sistema de preparo do solo tradicional adotado pelos agricultores consiste no revolvimento total do solo com arado de tração animal. Nos primeiros estádios da cultura o solo é mantido livre de plantas competidoras, seja por meios mecânicos ou com herbicidas. Após a colheita, alguns agricultores revolvem novamente o solo para plantio de culturas de inverno. Associado a essas práticas está vinculado o período de maior erosividade das chuvas, que coincide com a época de preparo do solo e o início do estabelecimento das culturas. Assim, os meses críticos em relação à cobertura de solo são de agosto a dezembro e de maio a julho (Merten \& Minella, 2003).

\section{Amostragem e análises das fontes e dos sedi- mentos em suspensão}

A coleta das amostras nas fontes foi realizada nos locais onde a erosão hídrica é evidente: lavouras, estradas e rede fluvial. A rede fluvial corresponde aos canais de escoamento natural da bacia. Foi estabelecida uma malha de amostragem representativa da variabilidade em cada fonte. Na bacia de Agudo foram

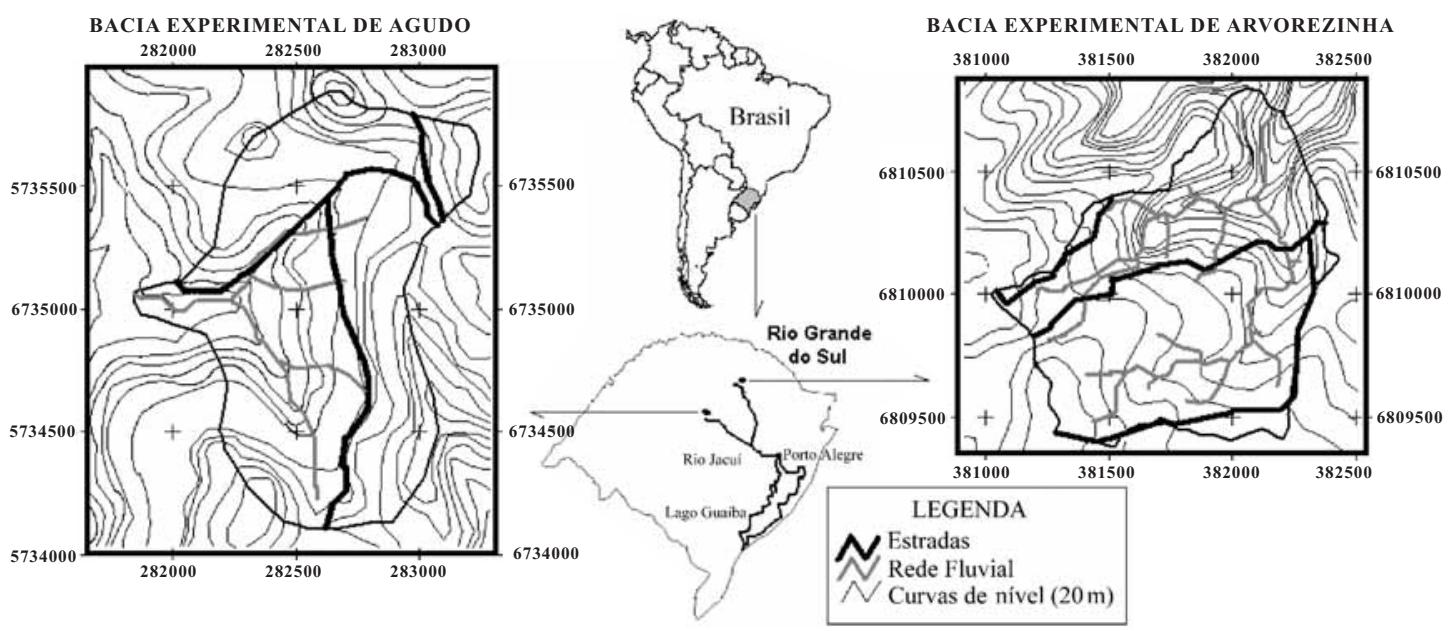

Figura 1. Localização das bacias de Agudo e Arvorezinha. 
coletadas 20 amostras, sendo oito nas lavouras, seis nas estradas e seis na rede fluvial. Na bacia de Arvorezinha foram coletadas 28 amostras, sendo 12 nas lavouras, 8 nas estradas e 8 na rede fluvial. Para cada ponto de amostragem foram coletadas 10 subamostras $(0,5 \mathrm{~kg}$ na profundidade de $0-5 \mathrm{~cm})$. As amostras foram secas ao ar e divididas, com o uso de um quarteador; sendo uma parte enviada para análises químicas e a outra para análises físicas.

O monitoramento dos sedimentos em suspensão ocorreu nos eventos mais significativos do período de abril de 2003 a junho de 2004. A amostragem dos sedimentos em suspensão deve fornecer uma quantidade mínima de 40 g de material para realização das análises físicas e químicas. Para obter a quantidade necessária, precisa-se de, aproximadamente, $40 \mathrm{~L}$ de amostra do escoamento que foi coletado no exutório da bacia. Para coleta dessas amostras foi necessário adaptar um amostrador que coleta um volume adequado do escoamento, de modo que permitisse concentrar sedimentos suficientes para as análises. O amostrador desenvolvido é semelhante ao amostrador de nível ascendente (FISP, 1964), para possibilitar a coleta da amostra integrada em profundidade (ou seja, todo o perfil da lâmina de água). As principais características do amostrador são: (a) coleta um volume de amostra suficiente para as análises químicas e físicas; (b) coleta automática e continuamente a amostra durante a subida e a descida da onda de cheia; (c) integra o perfil vertical do canal de drenagem; (d) os bocais de coleta estão conectados por mangueiras a um galão de $40 \mathrm{~L}$; (e) o espaçamento entre os bocais de coleta é de $7 \mathrm{~cm}$; e (f) o primeiro bocal está localizado a $40 \mathrm{~cm}$ do fundo do canal, o que possibilita a coleta dos eventos de média e grande magnitude. Após cada evento, os técnicos das bacias retiram as amostras do galão e enviam para o laboratório. As amostras foram centrifugadas numa centrífuga de fluxo contínuo (Minella, 2003) e os sedimentos armazenados a $4{ }^{\circ} \mathrm{C}$, para posterior análise.

O material coletado nas fontes e os sedimentos em suspensão foram peneirados em malha de $150 \mu \mathrm{m}$ e encaminhados ao laboratório de análises químicas. $\mathrm{O}$ limite de $150 \mu \mathrm{m}$ foi escolhido para diminuir as incertezas relacionadas com o efeito diluição das partículas de areia e considerar os agregados de argila que estão em suspensão. Para a análise física, as amostras do material das fontes e dos sedimentos em suspensão foram peneiradas na malha de $500 \mu \mathrm{m}$, submetendo-as a análise em granulômetro laser (Muggler et al., 1997). Esse equipamento estima o tamanho das partículas com base numa esfera correspondente de mesmo volume. A faixa granulométrica obtida é entre 0,04 e $500 \mu \mathrm{m}$, com discretização de 100 classes.

As análises químicas foram realizadas no Laboratório de Química do Solo da Universidade Federal de Santa Maria. Foram determinados os teores totais de $\mathrm{P}, \mathrm{K}, \mathrm{Ca}, \mathrm{Na}, \mathrm{Mg}, \mathrm{Cu}, \mathrm{Pb}, \mathrm{Cr}, \mathrm{Co}, \mathrm{Zn}$,
$\mathrm{Ni}, \mathrm{Fe}, \mathrm{Mn}$ e Al, com extração por digestão ácida (EPA, 1996). A concentração de $P$ no extrato foi quantificada por espectrofotocolorimetria; as de $\mathrm{K}$ e $\mathrm{Na}$, por espectrometria de emissão de chama; e a dos demais elementos, por espectrometria de absorção atômica.

Alguns dos elementos químicos utilizados para discriminar as três fontes potenciais de sedimentos refletem as práticas agrícolas da bacia. Uma importante fração do $\mathrm{P}$ e do $\mathrm{K}$, presente nos solos e nos sedimentos erodidos, está fortemente associada aos minerais de argila. Sua variabilidade entre as fontes está associada com as práticas de fertilização dos solos. A aplicação de fertilizantes químicos e orgânicos nas áreas de cultivo resulta em maior concentração de $\mathrm{P}$ e de $\mathrm{K}$ nas áreas de lavouras, em concentração baixa nas estradas que são construídas com material de subsolo e em concentração intermediária na rede fluvial. Tal como o $\mathrm{P}$ e o K, as concentrações de Ca e de Na também são relacionadas com a variabilidade do uso do solo na bacia. O Ca é aplicado nas lavouras como calcário, para regular o pH. O Na é encontrado em maior concentração em lavouras antigas devido aos efeitos acumulativos de fertilizações com salitre-do-chile $\left(\mathrm{NaNO}_{3}\right)$, que são muito utilizadas no cultivo de fumo, por serem facilmente assimiláveis pelas plantas.

As concentrações de metais pesados, como Zn e $\mathrm{Cu}$, também podem apresentar concentrações diferentes entre as fontes de sedimentos; além disso, esses elementos podem ser considerados bons traçadores, particularmente por seu comportamento conservativo no ambiente. Os metais pesados são largamente utilizados na agricultura como fertilizantes, bactericidas e fungicidas (Adriano, 2001). Alguns metais pesados também estão associados a combustíveis, óleos e graxas presentes nos veículos, os quais podem ser adicionados nas estradas. Dessa forma, a concentração de metais pesados nos solos das fontes permite a diferenciação, principalmente, das áreas de lavoura e estradas.

Os teores de Fe e Mn associados aos óxidos pedogênicos foram determinados por extração com ditionito-citrato-bicarbonato de $\mathrm{Na}$ e associados aos óxidos pedogênicos de baixa cristalinidade, por extração com oxalato de amônio ácido no escuro (McKeague \& Day, 1966; Inda Junior, 2002). A variabilidade da concentração desses elementos está associada com o fenômeno de oxirredução dos solos. A condição de maior umidade do solo (rede fluvial e várzeas) pode afetar as suas concentrações, devido ao efeito do redox e à sua mobilidade na forma reduzida.

\section{Análises estatísticas}

A primeira etapa da análise estatística constitui em estabelecer o conjunto de variáveis com capacidade de discriminar as fontes. Para isso, foram utilizados dois testes estatísticos: (a) o teste não-paramétrico Kruskal-Wallis (H); e (b) uma função discriminante multivariada. $\mathrm{O}$ teste $H$ permite testar a hipótese 
nula de que as fontes são provenientes de uma mesma população. Neste teste foram definidos quais elementos químicos apresentam diferença estatística entre as três fontes de sedimentos, os quais podem ser utilizados como variáveis traçadoras. $\mathrm{O}$ teste foi aplicado para cada variável, verificando sua capacidade individual em discriminar as fontes (Equação 1).

$$
H=\frac{12}{n(n+1)} \sum_{s=1}^{k} \frac{R_{s}^{2}}{n_{l}}-3(n+1)
$$

em que $R_{s}$ se refere à soma dos "ranks" ocupados pela fonte $s$, sendo $n_{l}$ o número de observações da fonte $s$; $n$, a soma dos $n_{1}$ 's; e $k$, o número de fontes.

O segundo teste é uma função discriminante multivariada que determina o número mínimo de variáveis que maximiza a discriminação das fontes. O método é baseado no parâmetro Wilks' Lambda $\left(\Lambda^{*}\right)$ da análise de variância e o critério utilizado pelo modelo estatístico é a minimização de $\Lambda^{*}$ (Equação 2). Não existe capacidade discriminante (as fontes são consideradas iguais) do conjunto de variáveis selecionadas quando $\Lambda^{*}$ é próximo de 1 . Valores próximos de 0 ocorrem quando a variabilidade dentro do grupo é pequena em comparação com a variabilidade total, isto é, em a maior parte da variabilidade total pode ser atribuída à diferença entre os grupos.

$$
\Lambda^{*}=\frac{|W|}{|B+W|}
$$

em que $|\mathrm{W}|$ é o determinante da matriz da soma dos quadrados devido ao erro, enquanto $|\mathrm{B}+\mathrm{W}|$ representa o determinante da matriz da soma dos quadrados totais.

A segunda etapa da análise estatística consistiu da classificação das amostras de sedimentos em suspensão. O método supõe que os sedimentos em suspensão são uma mistura de material das fontes e que a comparação da concentração dos elementos das fontes com os elementos dos sedimentos em suspensão pode definir a contribuição de cada fonte. A equação 3 descreve a relação matemática entre as proporções das fontes e as variáveis das fontes e dos sedimentos em suspensão (Walling \& Woodward, 1995).

$$
\begin{gathered}
y_{i}=\sum_{s=1}^{n} a_{i s} P_{s} \\
(\mathrm{~s}=1,2, \ldots, \mathrm{n}) \text { e }(\mathrm{i}=1,2, \ldots, \mathrm{m})
\end{gathered}
$$

em que $y_{i}$ é o valor da variável $i$ obtida no sedimento em suspensão, $a_{i s}$ são os coeficientes do modelo linear (concentração do elemento químico ina fonte $s$ ) e $P_{s}$ é a proporção da massa da fonte $s$, que podem ser apresentados como um conjunto de funções lineares de $m$ variáveis e $n$ fontes.

Para determinar os valores de $P$ foi utilizada uma função objetivo (Walling \& Woodward, 1995). A solução é encontrada por meio de um processo iterativo, com o objetivo de minimizar o valor de $R$
(Equação 4). No processo de minimização, os valores de $P$ devem estar sujeitos a duas restrições: devem ser maiores ou iguais a zero e menores ou iguais a 1 ; e a soma dos $P$ deve ser igual a 1 . O modelo foi executado no programa Matlabß.

$$
R=\sum_{i=1}^{m}\left\{\left(C_{i}-\left(\sum_{s=1}^{n} P_{s} C_{s i} Z_{s}\right)\right) / C_{i}\right\}^{2}
$$

em que $m$ é o número de elementos químicos selecionados como variáveis traçadoras; $n$, o número de fontes; $C_{i}$, a concentração da variável traçadora $i$ na amostra de sedimento em suspensão; $P_{s}$, a proporção de contribuição da fonte $s$; $C_{s i}$, o valor médio da variável traçadora $i$ obtido na fonte $s$; e $Z_{s}$, o fator de correção granulométrica da amostra da fonte $s$.

A etapa final do método de identificação das fontes foi verificar se o processo de otimização da equação 4 forneceu resultados aceitáveis da contribuição relativa de sedimentos de cada fonte. A avaliação dos resultados foi feita comparando a concentração química dos elementos utilizados (variáveis traçadoras) nos sedimentos em suspensão e o valor predito pelo modelo, com base na proporção calculada para cada fonte. Com os valores do erro relativo de cada variável, foi calculada uma média (ERM) para providenciar um valor único associado a cada amostra de sedimentos em suspensão (Equação 5).

$$
E R M=\sum_{i=1}^{m}\left\{\left(C_{i}-\left(\sum_{s=1}^{n} P_{s} C_{s i}\right)\right) / C_{i}\right\} / m
$$

Segundo Walling \& Collins (2000), valores menores que $15 \%$ para a equação 5 indicam que o modelo encontrou uma solução viável dos valores de $P_{s}$ (contribuições relativas de cada fonte) provenientes do procedimento de minimização da equação 4 .

\section{RESULTADOS E DISCUSSÃO}

\section{Análise discriminante}

No quadro 1 estão apresentadas as concentrações médias dos elementos selecionados pelo teste estatístico $H$ (Kruskal-Wallis), que determinou quais elementos apresentam diferença estatística entre as três fontes para uma probabilidade igual ou maior que $90 \%$. No quadro 2 encontra-se o resultado do teste estatístico, mostrando o valor de $\mathrm{H}$ calculado para cada variável e a probabilidade correspondente de que o elemento tem capacidade discriminante entre as três fontes.

A análise individual das variáveis (teste $\mathrm{H}$ ou Kruskal-Wallis) mostrou que $70 \%$ dos elementos previamente escolhidos apresentam capacidade discriminante entre as fontes. Na bacia de Agudo, o número de elementos selecionados na análise discriminante foi maior do que na bacia de 
Arvorezinha: 13 e 10 elementos, respectivamente. variáveis nas duas bacias é semelhante: 98,0\% em Entretanto, a capacidade de discriminação das Agudo e 99,8 \% em Arvorezinha.

Quadro 1. Variáveis selecionadas pelo teste de discriminação Kruskal-Wallis

\begin{tabular}{|c|c|c|c|c|c|c|}
\hline \multirow{2}{*}{ Variável } & \multicolumn{3}{|c|}{ Agudo } & \multicolumn{3}{|c|}{ Arvorezinha } \\
\hline & $\mathrm{CV}$ & $\mathbf{H}$ & $p$ & $\mathrm{CV}$ & $\mathbf{H}$ & $p$ \\
\hline Total & $\%$ & & $\%$ & $\%$ & & $\%$ \\
\hline Zinco $(\mathrm{Zn})$ & 18,5 & 9,1 & 98,0 & 13,8 & 4,1 & 94,0 \\
\hline Cobre $(\mathrm{Cu})$ & 43,9 & 10,0 & 98,0 & 13,4 & 12,3 & 99,8 \\
\hline Manganês (Mn) & 15,9 & 10,2 & 98,0 & 39,6 & 15,9 & 99,9 \\
\hline Ferro $(\mathrm{Fe})$ & 27,0 & 9,8 & 98,0 & $\mathrm{x}$ & $\mathrm{x}$ & $\mathrm{x}$ \\
\hline Fósforo (P) & 45,1 & 8,7 & 97,0 & 39,3 & 18,7 & 99,9 \\
\hline Cálcio (Ca) & 31,9 & 10,7 & 98,0 & 45,8 & 12,6 & 99,8 \\
\hline Magnésio (Mg) & 29,0 & 10,3 & 98,0 & $\mathrm{x}$ & $\mathrm{x}$ & $\mathrm{x}$ \\
\hline Potássio (K) & 32,1 & 12,0 & 99,0 & 35,0 & 17,2 & 99,9 \\
\hline Sódio (Na) & 34,6 & 17,7 & 99,9 & 34,3 & 11,5 & 99,7 \\
\hline \multicolumn{7}{|l|}{ Óxidos } \\
\hline Ferro $\left(\mathrm{Fe}_{\mathrm{dit}}\right)$ & 31,5 & 6,2 & 96,0 & $\mathrm{x}$ & $\mathrm{x}$ & $\mathrm{x}$ \\
\hline Manganês $\left(\mathrm{Mn}_{\mathrm{dit}}\right)$ & 32,2 & 6,5 & 97,0 & 52,5 & 12,1 & 99,8 \\
\hline Ferro $\left(\mathrm{Fe}_{\text {oxal }}\right)$ & 35,8 & 3,7 & 95,0 & 64,7 & 14,5 & 99,9 \\
\hline Manganês $\left(\mathrm{Mn}_{\text {oxal }}\right)$ & 25,8 & 14,4 & 99,5 & 56,3 & 12,1 & 98,0 \\
\hline
\end{tabular}

\section{Quadro 2. Concentrações dos elementos químicos utilizados como traçadores naturais, em cada fonte}

\begin{tabular}{|c|c|c|c|c|c|c|}
\hline \multirow{2}{*}{ Variável } & \multicolumn{3}{|c|}{ Arvorezinha } & \multicolumn{3}{|c|}{ Agudo } \\
\hline & Lavoura & Estrada & Rede fluvial & Lavoura & Estrada & Rede fluvial \\
\hline Total & \multicolumn{3}{|c|}{$\mathrm{mg} \mathrm{L}^{-1}$} & \multicolumn{3}{|c|}{$\mathrm{mg} \mathrm{L}^{-1}$} \\
\hline Zinco & 3,28 & 3,17 & 2,87 & 4,25 & 3,24 & 3,06 \\
\hline Cobre & 0,26 & 0,30 & 0,23 & 1,79 & 0,91 & 0,77 \\
\hline Manganês & 328 & 145 & 238 & 343 & 297 & 317 \\
\hline Ferro & 298.561 & 307.395 & 274.804 & 602.914 & 375.832 & 337.789 \\
\hline Fósforo & 783 & 397 & 330 & 1.277 & 532 & 550 \\
\hline Cálcio & 2.178 & 1.001 & 2.091 & 2.952 & 2.509 & 2.463 \\
\hline Magnésio & 606 & 476 & 560 & 1.527 & 1.048 & 1.102 \\
\hline Potássio & 2.185 & 1.008 & 1.219 & 3.779 & 1.752 & 2.402 \\
\hline Sódio & 716 & 411 & 466 & 1.241 & 267 & 240 \\
\hline \multicolumn{7}{|l|}{ Óxidos } \\
\hline Ferro $\left(\mathrm{Fe}_{\mathrm{dit}}\right)$ & 18.328 & 17.850 & 23.500 & 2.263 & 1.322 & 1.450 \\
\hline Manganês (Mndit) & 1.443 & 636 & 878 & 33.407 & 15.454 & 19.478 \\
\hline Ferro $\left(\mathrm{Fe}_{\text {oxal }}\right)$ & 3.174 & 1.223 & 4.326 & 1.300 & 608 & 650 \\
\hline Manganês $\left(\mathrm{Mn}_{\text {oxal }}\right)$ & 702 & 211 & 447 & 2.591 & 1.393 & 4.087 \\
\hline
\end{tabular}


Segundo Horowitz (1991), os metais de transição apresentam boa conservatividade; das 12 variáveis analisadas no presente estudo $(\mathrm{Co}, \mathrm{Pb}, \mathrm{Cr}, \mathrm{Cu}, \mathrm{Zn}$, $\mathrm{Ni}, \mathrm{Fe}, \mathrm{Fe}_{\text {dit }}, \mathrm{Fe}_{\text {oxal }}, \mathrm{Mn}, \mathrm{Mn}_{\text {dit }}$ e $\left.\mathrm{Mn}_{\text {oxal }}\right)$, oito apresentaram capacidade discriminante entre as fontes $\left(\mathrm{Cu}, \mathrm{Zn}, \mathrm{Fe}, \mathrm{Fe}_{\text {dit }}, \mathrm{Fe}_{\text {oxal }}, \mathrm{Mn}, \mathrm{Mn}_{\text {dit }}\right.$ e $\left.\mathrm{Mn}_{\text {oxal }}\right)$. As variáveis $\mathrm{Pb}, \mathrm{Cr}, \mathrm{Cu}$ e $\mathrm{Ni}$ não mostraram capacidade discriminante entre as fontes, até porque os valores estão muito próximos do limite de detecção do aparelho e, por isso, foram retirados da análise.

$\mathrm{O}$ elemento $\mathrm{P}$ apresentou bom resultado como variável discriminante das fontes para as duas bacias. Esse elemento apresenta grande afinidade pelas partículas de argila e óxidos, o que o torna um ótimo traçador de sedimentos em suspensão. Sua concentração difere significativamente entre as fontes e está vinculada à característica da ocupação agrícola nas bacias. Nas áreas de lavoura é aplicada excessiva quantidade de adubos fosfatados, distinguindo facilmente essa fonte das demais. As estradas apresentam quantidade baixa de nutrientes como $\mathrm{Ca}, \mathrm{Mg}, \mathrm{Ke} \mathrm{P}$, uma vez que os sedimentos são provenientes do horizonte C, enquanto na rede fluvial são encontrados valores intermediários entre as lavouras e as estradas.

Na identificação de fontes de sedimentos grande número de variáveis químicas é desejado, pois podem distinguir mais processos efetivos nos diferentes tipos de erosão e, assim, diminuir as incertezas relacionadas com a possibilidade de enriquecimento ou depleção (Small et al., 2002). Entretanto, isso aumenta a dimensionalidade da análise, o que dificulta a análise de classificação (Johnson \& Wichern, 1992). Dessa forma, é importante otimizar o conjunto de elementos a ser utilizado, maximizando a discriminação com o menor número possível de variáveis. Os resultados da segunda etapa da análise discriminante multivariada, com o grupo de variáveis discriminantes para as duas bacias, estão apresentados no quadro 3 .

Os resultados (Quadro 3) mostram que para as duas bacias houve número suficiente de variáveis e com capacidade discriminante entre as fontes. Sete dos oito elementos selecionados são iguais nas duas bacias; os únicos elementos distintos entre as bacias foram Mg para Agudo e Fe para Arvorezinha. Isso pode estar relacionado com alguma diferença nos so- los das bacias ou nas práticas agrícolas de cultivo do fumo. Os elementos $\mathrm{Fe}_{\text {dit }}, \mathrm{Fe}_{\text {oxal }}$ e $\mathrm{Mn}_{\text {dit }}$ não foram estatisticamente significativos na minimização do parâmetro Wilks' Lambda. $\mathrm{O} \mathrm{Mn}_{\text {oxal }}$ foi estatisticamente significativo, mas apresenta forte correlação com o valor de Mn total e, por isso, foi excluído do conjunto pela função de discriminação.

Sendo o valor de $\Lambda^{*}$ definido como a proporção da variabilidade total das fontes devido ao erro, observase que o conjunto das oito variáveis reduziu esse valor para, aproximadamente, 7,7 e 7,6 \% para as bacias de Agudo e Arvorezinha, respectivamente. Isso significa que o conjunto de elementos químicos selecionados explica, aproximadamente, 92,3 e 92,4 \% das diferenças químicas entre as três fontes para as bacias de Agudo e Arvorezinha, respectivamente.

\section{Análise de classificação}

No quadro 4 encontra-se a média das contribuições relativas de cada fonte para os sedimentos em suspensão dos eventos monitorados entre junho de 2003 e junho de 2004 (oito eventos em Agudo e doze em Arvorezinha). O modelo de classificação mostrou-se eficiente na minimização da função objetiva, tendo em vista os erros médios serem menores que $15 \%$. A contribuição das fontes, para as duas bacias, apresenta o mesmo padrão de importância, com predomínio das lavouras, seguido pelas estradas e pela rede fluvial - esta com a menor contribuição.

No entanto, as duas bacias apresentam diferenças na magnitude de cada fonte e na variabilidade entre os períodos. Na bacia de Agudo, as lavouras contribuem mais em comparação com Arvorezinha, possivelmente pela maior declividade das áreas de lavouras e, conseqüentemente, maior capacidade de transferência dos sedimentos erodidos para a calha. Em vários locais desta bacia não há presença de ambiente ripário entre as lavouras e a rede fluvial. No caso de Arvorezinha, a inadequada alocação das estradas na bacia (no sentido das pendentes) e o grande comprimento destas favorecem o aumento da contribuição dessa fonte na produção de sedimentos da bacia. Pode-se afirmar que, proporcionalmente, a importância da erosão nas lavouras em Agudo é maior que em Arvorezinha.

\section{Quadro 3. Resumo da análise discriminante multivariada}

\begin{tabular}{ccccccccccc}
\hline \multirow{2}{*}{ Agudo } & \multicolumn{7}{c}{ Wilks' Lambda $\left(\Lambda^{(1)}\right)$ acumulado } \\
\cline { 2 - 5 } & $\mathbf{C a}$ & $\mathbf{P}$ & $\mathbf{K}$ & $\mathbf{Z n}$ & $\mathbf{C u}$ & $\mathbf{N a}$ & $\mathbf{M g}$ & $\mathbf{M n}$ \\
\hline$\Lambda^{(1)}$ & 0,155 & 0,127 & 0,116 & 0,090 & 0,084 & 0,079 & 0,078 & 0,077 \\
Arvorezinha & $\mathbf{P}$ & $\mathbf{C u}$ & $\mathbf{K}$ & $\mathbf{C a}$ & $\mathbf{M n}$ & $\mathbf{N a}$ & $\mathbf{Z n}$ & $\mathbf{F e}$ & 0,076 \\
$\Lambda^{(1)}$ & 0,129 & 0,084 & 0,092 & 0,097 & 0,092 & 0,083 & 0,078 & 0,076 \\
\hline
\end{tabular}

(1) Número de variáveis no modelo $=8$; Agrupamento $=$ três fontes. 
A análise do coeficiente de variação, considerando as diferenças na declividade das bacias, também sugere que na bacia de Agudo, mais do que na de Arvorezinha, prevalece uma condição que favorece a transferência de sedimentos das lavouras para a rede fluvial. No caso da bacia de Arvorezinha, é provável que os sedimentos mobilizados pelo processo erosivo nas lavouras permaneçam tempo maior em depósitos próximo e dentro da rede fluvial, o que ocasionaria transferência mais lenta através da rede fluvial, em comparação com Agudo. No caso dos sedimentos erodidos nas estradas, não há muitos fatores que impedem a transferência para dentro da calha. Os sedimentos erodidos na rede fluvial são dependentes das condições de escoamento e da declividade do canal, além do aporte de sedimentos provenientes das lavouras e estradas, que afeta a energia do escoamento.

A variabilidade temporal das contribuições das lavouras está vinculada com o calendário agrícola de cultivo do fumo e com a característica climática da região. Em geral, o preparo do solo inicia-se em agosto, cuja cobertura vegetal é mantida baixa até novembro, quando as plantas atingem porte maior. No entanto, mesmo antes de atingir o máximo crescimento da cultura do fumo, inicia-se a colheita das folhas, expondo progressivamente o solo à ação das chuvas, culminando no final da colheita (início de janeiro em Agudo e meados de fevereiro em Arvorezinha). Alguns agricultores voltam a arar o terreno nesse período para o plantio de culturas de inverno. Isso confirma os resultados da contribuição relativa de cada fonte $(P)$ calculados pelo modelo (Equações 3 a 5), que indicou a maior contribuição das lavouras de junho a outubro e de março a junho.

Quanto aos aspectos climáticos, três períodos distintos são importantes para compreender a variabilidade da produção de sedimentos. O primeiro é o período de maior erosividade das chuvas, que ocorre, em geral, nos meses de setembro e outubro. O segundo é aquele dos meses úmidos e frios (junho a agosto), quando, devido à baixa evapotranspiração, os solos mantêm-se encharcados e com grande potencial para gerar escoamento superficial. Eventos de significativa precipitação nesse período transportam grande quantidade de sedimentos. O terceiro período compreende os meses de verão. Neste período ocorre número menor de eventos significativos de chuva-vazão com produção de sedimentos, devido à maior freqüência de estiagens, menor umidade do solo e maior capacidade de retenção do volume de água precipitado.

\section{Conseqüências para o manejo e controle dos sedimentos na escala de bacia}

Considerando que, em geral, recursos financeiros disponíveis para manejo integrado de bacias são escassos, determinar a contribuição relativa de cada fonte de sedimentos ou poluente é, no mínimo, economicamente vantajoso (Walling, 2005). Com esse tipo de informação, podem-se programar as atividades e os gastos no controle da erosão e da produção de sedimentos conforme a importância de cada fonte. Nas bacias analisadas, essas considerações têm aplicação direta, posto que, apesar de o impacto das áreas de lavoura ser evidente sobre a produção de sedimentos, as estradas e a rede fluvial também contribuem de forma significativa para produção de sedimentos.

As estradas, que ocupam uma área 25 vezes menor que a das lavouras, contribuem com um terço da produção de sedimentos. As principais causas desse impacto são: falta de planejamento da alocação das estradas na bacia (declividade acentuada e grande comprimento); composição inadequada do material; baixa freqüência de manutenção; ligação direta entre as estradas e a rede fluvial; e ligação direta entre as lavouras e as estradas. Nesse caso, o melhor planejamento na alocação das estradas e na sua manutenção teria grande impacto na diminuição da produção de sedimentos nas duas bacias estudadas.

No caso da rede fluvial, a grande energia do escoamento que ocorre durante as cheias, associada à pequena conservação, ou ausência total da mata ciliar,

\section{Quadro 4. Resultado da análise de classificação das amostras de sedimentos em suspensão}

\begin{tabular}{|c|c|c|c|c|c|c|}
\hline \multirow{2}{*}{ Mês } & \multicolumn{3}{|c|}{ Agudo } & \multicolumn{3}{|c|}{ Arvorezinha } \\
\hline & Lavoura & Estrada & Rede fluvial & Lavoura & Estrada & Rede fluvial \\
\hline Janeiro-Outubro & 67,5 & 29,1 & 3,4 & 56,9 & 31,4 & 11,8 \\
\hline Novembro-Fevereiro & 60,0 & 36,2 & 3,8 & 51,8 & 40,4 & 7,8 \\
\hline Março-Junho & 77,5 & 19,1 & 3,5 & 57,8 & 41,0 & 1,3 \\
\hline Média (\%) & 68,3 & 28,1 & 3,6 & 55,5 & 37,6 & 6,9 \\
\hline $\mathrm{CV}(\%)$ & 12,8 & 30,6 & 6,6 & 5,8 & 14,3 & 76,5 \\
\hline $\operatorname{ERM}(\%)^{(1)}$ & 7,6 & 11,4 & 6,3 & 5,9 & 13,5 & 5,7 \\
\hline
\end{tabular}

(1) Erro relativo médio. 
favorece que as margens sejam facilmente erodidas, devido à menor resistência à ação cisalhante do escoamento e à menor rugosidade hidráulica decorrente da ausência da vegetação. Segundo Lowrance et al. (2002), a implementação de áreas de preservação permanente nas áreas ciliares pode ser extremamente eficiente para estabilização das margens, resistência ao fluxo da onda de cheia, filtragem de poluentes e interceptação dos sedimentos provenientes das lavouras.

\section{CONCLUSÕES}

O trabalho apresenta três conclusões para a análise da produção de sedimentos em regiões do planalto basáltico do Rio Grande do Sul.

1. O método de identificação das fontes de sedimentos foi eficiente na determinação das principais fontes (lavouras, estradas e rede fluvial). Isso permitiu quantificar as suas contribuições relativas para produção total de sedimentos das bacias. Na fase de discriminação das fontes, foi determinado um número razoável de elementos químicos que puderam ser utilizados como variáveis com potencial traçador. $\mathrm{Na}$ fase de classificação, o modelo de identificação determinou corretamente a contribuição de cada fonte; os erros médios relativos foram menores que os valores críticos recomendados.

2. As duas bacias apresentaram padrão semelhante de contribuição das fontes. Na bacia de Agudo, a contribuição das lavouras foi de $68,3 \%$; das estradas, de $28,1 \%$; e da rede fluvial, de $3,6 \%$. Na bacia de Arvorezinha, a contribuição das lavouras foi de $55,5 \%$; das estradas, de $37,6 \%$; e da rede fluvial, de $6,9 \%$.

3. As lavouras são as principais fontes de sedimentos. Programas de capacitação e implementação de práticas de manejo conservacionista dos solos são essenciais. As estradas e a rede fluvial também são fontes importantes de sedimentos; a correta alocação e manutenção das estradas e a preservação das áreas ciliares têm significativo impacto na redução da erosão e da produção de sedimentos em projetos de conservação e manejo de solos em bacias hidrográficas, além de serem fundamentais para garantir a qualidade do ecossistema fluvial.

\section{AGRADECIMENTOS}

Os autores agradecem aos técnicos Adair Giongo, Ademir Giongo e Antonio V. Britzke pelos trabalhos de campo; à equipe do Laboratório de Análise Química do Departamento de Solos da UFSM; ao Conselho Nacional de Desenvolvimento Científico e Tecnológico (CNPq); à Fundação de Amparo a Pesquisa do Rio Grande do Sul (FAPERGS) e ao Sindicato das
Empresas Fumageiras (SINDIFUMO), pelo apoio financeiro ao projeto; e à Elena Brett Metcalf, pela tradução do resumo.

\section{LITERATURA CITADA}

ADRIANO, D.C. Trace elements in terrestrial environments: biogeochemistry, bioavailability, and risks of metals. 2.ed. New York, Springer, 2001. 867p.

COLLINS, A.L. \& WALLING, D.E. Selecting fingerprint properties for discriminating potential suspended sediment sources in river basins. J. Hydrol., 261:218-244, 2002.

COLLINS, A.L.; WALLING, D.E. \& LEEKS, G.J.L. Source type ascription for fluvial suspended sediment based on a quantitative composite fingerprinting technique. Catena, 29:1-27, 1997.

FEDERAL INTERAGENCY SEDIMENTATION PROJECT FISP. The single-stage sampler for suspended sediment. Minneapolis, St. Anthony Falls Hydraulics Laboratory, Inter-Agency, 1961. p.1-105. (Report, 13)

HOROWITZ, A.J. A primer on sediment-trace element chemistry. 2.ed. Chelsea, Lewis. 1991. 136p.

INDA JUNIOR, A.V. Caracterização de goethita e hematita em solos poligênicos. Porto Alegre, Universidade Federal do Rio Grande do Sul, 2002. 126p. (Tese de Doutorado).

JOHNSON, R.A. \& WICHERN, D.W. Applied multivariate statistical analysis. London, Prentice-Hall International, 1992. 642p.

LOWRANCE, R.; DABNEY, S. \& SCHULTZ, R. Improving soil and water quality with conservation buffers. J. Soil Water Conserv., 57:36-43, 2002.

MCKEAGUE, J.A. \& DAY, J.H. Dithionite and oxalateextractable $\mathrm{Fe}$ and $\mathrm{Al}$ as aids in differentiating various classes of soil. Can. J. Soil Sci., 46:13-22, 1966.

MERTEN, G.H. \& MINELLA, J.P.G. Projeto de monitoramento ambiental de microbacias hidrográficas - RS-RURAL subprojeto 7. Porto Alegre, IPH-UFRGS, 2003. 89p.

MINELLA, J.P.G. Identificação de fontes de sedimentos em uma pequena bacia rural. Porto Alegre, Universidade Federal do Rio Grande do Sul, 2003. 90p. (Tese de Mestrado)

MUGGLER, C.C.; PAPE T. \& BUURMAN, P. Laser grain-size determination in soil genetic studies. 2. Clay content, clay formation, and aggregation in some Brazilian oxisols. Soil Sci., 162:219-228, 1997.

RUSSELL, M.A.; WALLING, D.E. \& HODGKINSON, R.A. Suspend sediment sources in two small lowland agricultural catchments in the UK. J. Hydrol., 252:1-24, 2001.

SMALL, I.F.; ROWAN, J.S. \& FRANKS, S.W. Quantitative sediment fingerprinting using a Bayesian uncertainty estimation framework. In: DYER, F.J.; THOMS, M.C. \& OLLEY, J.M., eds. The structure, function, and management implications of fluvial sedimentary. Wallingford, International Association of Hydrological Sciences, 2002. p.443-450. 
UNITED STATES ENVIRONMENTAL PROTECTION AGENCY - EPA. Method 3050-B. Acid digestion on sediments sludge's and soils. Washington, 1996.

WALLING, D.E. Tracing suspend sediment sources in catchments and river systems. Sci. Total Environ., 344:159-184, 2005.

WALLING, D.E. \& COLLINS, A.L. Integrated assessment of catchment sediment budgets: A technical manual. Exeter, Department for International Development, 2000. 168 .
WALLING, D.E. \& WOODWARD, J.C. Tracing sources of suspend sediment in river basins: A case study of the River Culm, Devon, UK. Marine Freshwater Res.,46:327336, 1995.

WALLING, D.E.; HE, Q. \& WHELAN, P.A. Using Cs-137 measurements to validate the application of the AGNPS and ANSWERS erosion and sediment yield models in two small Devon catchments. Soil Till. Res., 69:27:43, 2003.

YU, L. \& OLDFIELD, F. A multivariate mixing model for identifying sediment source from magnetic measurements. Quaternary Res., 32:168-181, 1989. 